University of Wollongong

Research Online

Faculty of Engineering and Information

Faculty of Engineering and Information

Sciences - Papers: Part A

Sciences

2013

Effects of long-distance walking on socket-limb interface pressure, tactile sensitivity and subjective perceptions of trans-tibial amputees

L F. Yeung

The Hong Kong Polytechnic University

Aaron Leung

The Hong Kong Polytechnic University

Ming Zhang

The Hong Kong Polytechnic University

Winson Lee

University of Wollongong, ccwlee@uow.edu.au

Follow this and additional works at: https://ro.uow.edu.au/eispapers

Part of the Engineering Commons, and the Science and Technology Studies Commons

Research Online is the open access institutional repository for the University of Wollongong. For further information contact the UOW Library: research-pubs@uow.edu.au 


\title{
Effects of long-distance walking on socket-limb interface pressure, tactile sensitivity and subjective perceptions of trans-tibial amputees
}

\begin{abstract}
PURPOSE: Many trans-tibial amputees could not tolerate long-distance walking. Lack of walking could explain for the increased cardiovascular diseases mortality rate. This study investigated the effects of long-distance walking (LDW) on socket-limb interface pressure, tactile sensitivity of the residual limb, and subjective feedbacks, which potentially identified the difficulties in LDW. METHOD: Five male unilateral trans-tibial amputees walked on a level treadmill for a total of one hour at comfortable speed. Tactile sensitivity of the residual limb and socket-limb interface pressure during over-ground walking were measured before and after the treadmill walking. Modified Prosthesis Evaluation Questionnaires were also administered. RESULTS: After the treadmill walking, the socket-limb interface pressure and the tactile sensitivity at the popliteal depression area were significantly reduced. This corresponds well with the questionnaire results showing that the level of discomfort and pain of the residual limb did not increase. The questionnaire revealed that there were significant increases in fatigue level at the sound-side plantar flexors, which could lead to impaired dynamic stability. CONCLUSIONS: Fatigue of sound-side plantarflexor was the main difficulty faced by the five subjects when walking long-distances. This finding might imply the importance of refining prosthetic components and rehabilitation protocols in reducing the muscle fatigue.
\end{abstract}

\section{Keywords}

long-distance, subjective, perceptions, trans-tibial, walking, socket-limb, interface, pressure, tactile, effects, sensitivity, amputees

\section{Disciplines \\ Engineering | Science and Technology Studies}

\section{Publication Details}

Yeung, L. F., Leung, A. K. L., Zhang, M. \& Lee, W. C. C. (2013). Effects of long-distance walking on socketlimb interface pressure, tactile sensitivity and subjective perceptions of trans-tibial amputees. Disability and Rehabilitation, 35 (11), 888-893. 
Yeung LF, Leung AKL, Zhang M, *Lee WCC. Effects of long-distance walking on socket-limb interface pressure, tactile sensitivity and subjective perceptions of trans-tibial amputees. Disability and Rehabilitation 2013, 35,888-893. *Corresponding author.

\title{
Effects of long-distance walking on socket-limb interface pressure, tactile sensitivity and subjective perceptions of trans-tibial amputees
}

\author{
L.F. Yeung, Aaron K.L. Leung, Ming Zhang, Winson C.C. Lee*
}

Interdisciplinary Division of Biomedical Engineering, The Hong Kong Polytechnic University,

${ }^{*}$ Corresponding author

Dr. Winson Lee

Interdisciplinary Division of Biomedical Engineering

The Hong Kong Polytechnic University,

Hung Hom, Kowloon, Hong Kong,

E-mail: $\underline{\text { htwinson@inet.polyu.edu.hk }}$

Tel: +852 27664942

Fax: +852 23624365

Keywords: trans-tibial amputees, prosthesis, residual limb, Interface pressure, tactile sensitivity, long-distance walking, muscle fatigue, PEQ 


\section{Abstract (200 words)}

Purpose: Many trans-tibial amputees could not tolerate long distance walking. Lack of walking could explain for the increased cardiovascular diseases mortality rate. This study investigated the effects of long-distance walking on socket-limb interface pressure, tactile sensitivity of the residual limb, and subjective feedbacks, which potentially identified the difficulties in long-distance walking. Method: Five male unilateral trans-tibial amputees walked on a level treadmill for a total of one hour at comfortable speeds. Tactile sensitivity of the residual limb and socket-limb interface pressure during over-ground walking were measured before and after the treadmill walking. Modified Prosthesis Evaluation Questionnaires were also administered. Results: After the treadmill walking, the socket-limb interface pressure and the tactile sensitivity at the popliteal depression area were significantly reduced This corresponds well with the questionnaire results showing that the level of discomfort and pain of the residual limb did not increase. The questionnaire revealed that there were significant increases in fatigue level at the sound-side plantar flexors, which could lead to impaired dynamic stability. Conclusions: Fatigue of sound-side plantarflexors was the main difficulty faced by the five subjects when walking long-distances. This finding might imply the importance of refining prosthetic components and rehabilitation protocols in reducing the muscle fatigue.

Registered at HKU Clinical Trial Register (HKCTR-1370). http://www.HKClinicalTrials.com 


\section{Introduction}

Daily walking for over 30 minutes is recommended as it helps maintain good health [1]. Lower-limb amputees are recommended to walk for at least 3 hours a week [2]. Walking one hour or more daily could extend lifespan among older persons [3]. However, many amputees could not tolerate long-distance walking (LDW) [4,5]. Their lack of walking leads to a more sedentary lifestyle [6], which explains why their cardiovascular diseases mortality rate was higher than able people [7,8]. A previous study has reported the changes in gait patterns after LDW [9]. Further study on LDW was encouraged.

Information on prosthetic socket-residual limb interface pressure, residual limb tactile sensitivity, and amputees' subjective feedbacks could give additional clues in identifying the difficulties of LDW. Socket-limb interface pressure and tactile sensitivity are related to discomfort, pain, and protective responses of the residual limb to external pressure [10-13]. Changes in socket-limb interface pressure after long-distance walking could be caused by alterations in gait patterns after muscle fatigue $[9,14-16]$, as well as the changes in limb volume $[17,18]$ and tissue stiffness [19]. Socket-limb interface pressure at various areas of the residual limb has been studied in different conditions: interface friction [11,20], body movements [10,18,21], liner materials [22], prosthesis designs [21], and alignments [23]. It has also been studied using computational models as reviewed in [12,24]. Yet none of the previous studies examined the effects of LDW on dynamic interface pressure.

As pressure is repeatedly applied onto the residual limb upon walking, the tactile sensitivity and the mechanoreceptors responses could also be affected $[25,26]$. Tactile sensitivity can be determined using Semmes-Weinstein monofilaments, which measures the minimum pressure threshold that can be sensed by the skin [27]. The 
tactile sensitivity at the plantar foot has been extensively studied [28,29]. However, attention has not been paid on the residual limb. In addition, LDW might lead to poor socket fit and slippage of the residual limb against the socket, especially when the amputee sweated after walking. Muscle fatigue of the lower limb could reduce the walking stability and the body propulsion $[14,15]$.

The objective of this study was to investigate the effects of LDW on socketlimb interface pressure, tactile sensitivity of the residual limb, and the self-reported subjective feedbacks on socket fitting, interface conditions, dynamic stability, residual-limb comfort and pain feeling, and lower-limb muscle fatigue. Such analysis potentially identifies the problems of LDW for trans-tibial amputees.

\section{Method}

Subjects

Five male unilateral trans-tibial amputees, with mean age 52.6 years old (S.D.=4.3), height $169.3 \mathrm{~cm}$ (S.D.=1.7), and body weight $73.9 \mathrm{~kg}$ (S.D.=2.2), participated in this study with informed consent. They had amputation performed more than 9 years. Inclusion criteria were unilateral trans-tibial amputees, aged between 18 and 65, who were able to walk independently with their prostheses on a daily basis. Immature residual limb which might have large volume change was excluded. Subjects used their own prostheses with Patellar-Tendon-Bearing (PTB) sockets, Pe-Lite Liners, and solid-ankle-cushion-heel (SACH) prosthetic feet throughout the experiment. The prostheses were fitted properly as assessed by a professional prosthetist. Detailed subject characteristics were given in table 1. Ethical approval was obtained from The Human Subject Ethic Sub-committee of The Hong Kong Polytechnic University.

\section{Testing protocol}


Subjects performed two consecutive sessions of 30-minutes walking at their self-selected comfortable speed on a level treadmill. Dynamic socket-limb interface pressure was measured immediately (1) before the treadmill walking (baseline), (2) after the $1^{\text {st }}$ session of treadmill walking and (3) after the $2^{\text {nd }}$ session of treadmill walking. During the pressure measurement, subjects were instructed to walk overground along a straight 8-m walkway at their comfortable walking speed for five laps. Assessment of tactile sensitivity and subjective feedback were undertaken at the baseline and after the $2^{\text {nd }}$ session of treadmill walking. They were not undertaken after the $1^{\text {st }}$ session so as to minimize the time of rest of the subjects between the two sessions.

\section{Apparatus}

Dynamic socket-limb interface pressure measurement was monitored by FScan system (Tekscan). The film pressure sensors were adhered to the inner wall of the prosthetic liner at the anterior, posterior, anterior-medial, and anterior-lateral regions (figure 1). These allow pressure measurements at all major weight-bearing areas within the PTB prosthetic sockets [30], which included the patellar tendon, popliteal depression, anterior-medial tibia, and anterior-lateral tibia regions. The sampling frequency was $200 \mathrm{~Hz}$. New sensors were used for each subject. Prior to the measurements, each sensor was calibrated by applying a known compressive pressure of $200 \mathrm{kPa}$ uniformly onto the sensor. Linear relationships between sensor output and pressure within the range of $0-200 \mathrm{kPa}[21,31,32]$ were assumed. The sensors were pre-conditioned by performing a 30-cycle dynamic loading sequence. They were further pre-conditioned as the subjects were given five minutes to acclimatize to the instrumented prostheses before the measurements. The sensors were adhered to newly duplicated liners. To minimize drift and hysteresis owing to cyclic stress during 
treadmill walking, or any possible effects of sweat and changes in body temperature, the subjects walked with their own liners during treadmill walking, while using the new instrumented liners during pressure measurement. As PTB sockets have highly specified regions of undercut and relief areas [30], donning and doffing of the prosthetic sockets would not alter the residual limb position relative to the liners.

Tactile sensitivity at the residual limb was determined by measuring the pressure threshold using Semmes-Weinstein monofilaments (North Coast Medical, San Jose, CA, USA). Each filament had a specified diameter and a known buckling force. They were numbered such that each value was equal to $\log 10$ (10 x Force [mg]). The 1.65, 2.36, 2.83, 3.22, 3.61, 3.84, 4.08, 4.31, 4.56, 5.07, and 6.65 monofilaments were used in this study. A modified 4, 2 and 1 stepping algorithm [27-29] was used to evaluate the pressure threshold at four locations: patellar tendon, popliteal depression, anterior-medial tibia and anterior-lateral tibia. Subjects were informed by the examiner that a stimulus may or may not be applied. Each test began with the 3.84 filament. Depending on the response of the subject, the changes in stimulus intensity were made in four filament increments until the subject response changed and a turnaround point was reached. The changes in intensity were made in two filament increments until another turnaround point, at which all stimuli were presented in one filament increments. Tactile pressure threshold was determined to be the lightest filament that could be felt more than $50 \%$ of the time. For each location tested, the examiner performed a maximum of 25 trials with five null trials randomly placed throughout the algorithm. If the subject responded to more than one null stimulus at any given location, the test was halted and reinstructed.

Subjective feedback of the amputees was assessed using the modified version of the standard Prosthesis Evaluation Questionnaire (PEQ) [33] (table 2). Five 
questions were extracted from the PEQ. They questioned about socket fitting, interface conditions, dynamic stability, residual-limb comfort and pain feeling. An additional question was added to the questionnaire which asked the subjects the intensity of muscle fatigue perceived at the lower limb. At the end, subjects were requested to point at the locations of their lower limbs where the highest intensity of fatigue was perceived. A linear analog scale response format was used [34]. Each question was accompanied by a $10-\mathrm{cm}$ line bounded by two anchors denoting the extremes of possible answers. The most negative conditions were on the left and the most positive were on the right. The socket fitting score, interface conditions score, dynamic stability score, residual-limb comfort score, and pain feeling score, as well as lower-limb fatigue score were calculated based on the distance of the markings from the left anchors.

\section{Data analysis and statistics}

Socket-limb interface pressures were recorded in the middle 5-meter path of the 8-meter walkway. Peak interface pressures at each test region were normalized to the body weight at each sampling frame, and were averaged across each recorded walking step. The over-ground walking speed was determined from the time taken to complete the 8-m walkway. Peak interface pressures over a gait cycle, walking speeds, tactile pressure thresholds, and subjective feedback scores were averaged across subjects in each test condition. Bonferroni-adjusted t-tests for dependent samples were used to identify if there were significant differences in peak pressure between (1) the baseline and after 30-minutes treadmill walking, and (2) the baseline and after 1hour treadmill walking. Paired t-tests were performed to assess if the tactile pressure 
thresholds and the subjective feedback scores had significant differences between the baseline and after 1-hour treadmill walking. Significance level was set at $\mathrm{p}<0.05$.

\section{Results}

All five subjects were able to complete the two sessions of treadmill walking. The changes in walking speeds (table 3) were small and insignificant. As expected, high interface pressure was found at the patellar tendon, popliteal depression, anterior-medial tibia, and anterior-lateral tibia regions. Pressure was extremely low in other regions. Figure 2 showed the averaged peak interface pressures in stance phase of the gait cycle. There was a trend of reductions of interface pressure at the four high-pressure regions after treadmill walking (table 3). The average peak pressure reduced after 30-minutes, although statistical significance was not reached. After 1hour of treadmill walking, there were significant reductions in peak pressure at the popliteal depression $(-18.9 \%, \mathrm{p}=0.022)$ and the anterior-lateral tibia $(-20.9 \%$, $\mathrm{p}=0.007$ ), when compared with the baseline. The peak pressure at the patellar tendon reduced by $18.4 \%(p=0.10)$ and the anterior-medial tibia reduced by $20.5 \%(p=0.08)$.

After treadmill walking, the tactile pressure threshold at popliteal depression significantly increased by $15.6 \%(\mathrm{p}=0.04)$, indicating a significant reduction in tactile sensitivity at the region. Tactile pressure thresholds at other testing regions had no significant changes (table 4).

The fatigue scores of the questionnaire significantly decreased by $70 \%$ $(\mathrm{p}<0.001)$ (table 4$)$, indicating that the subjects perceived high intensity of muscle fatigue in the lower-limb. Four out of five subjects indicated that the highest intensity of fatigue was perceived at the sound-side plantar flexors. The remaining subject indicated that this was perceived equally at the sound-side plantar flexors and the 
prosthetic-side knee extensors. There were significant reductions in socket fitting score $(-9 \%, \mathrm{p}=0.03)$ and dynamic stability score $(-9 \%, \mathrm{p}=0.002)$. The differences in residual-limb comfort score, pain feeling score, and interface conditions score were small and insignificant.

\section{Discussion}

Lower-limb muscle fatigue could be the main reason for the trans-tibial amputees to have difficulties in LDW. The questionnaire showed that while there were small and insignificant changes in the interface conditions score, the residuallimb comfort score and the pain feeling score, there were large and significant decreases in the fatigue score after LDW. All subjects indicated that the fatigue originated from sound-side plantar flexors. The asymmetric gait pattern of trans-tibial amputees [35], who have longer stance time and greater ground reaction forces in sound side, could induce sound-side muscle fatigue more easily. The fatigue of the prosthetic-side knee extensors correspond well with a previous study which indicated increases in prosthetic-side knee joint flexion and moment during loading response in an attempt to facilitate the anterior rotation of the prosthetic shank after the soundside plantar flexors have fatigued [9]. Both the sound-side plantar flexors and the prosthetic-side knee extensors are important in providing walking stability. Thus the fatigue could be the cause of the significant reduction in the walking stability scores. It was consistent with a previous research which suggested that muscle fatigue after free-walking brought about impaired dynamic stability [15].

LDW did not cause problems in residual-limb comfort and pain feeling. The significant reduction of peak pressures at the weight-bearing areas of the residual limb reduced the probability of socket discomfort. The pressures were extremely low at regions other than the four weight-bearing regions, corresponding well with previous 
studies reviewed in [30]. The changes in the self-selected comfortable walking speed after LDW were very small, which could not be the causes of the reductions in peak interface pressures. The reductions could be caused by further shifting of the body weight to the sound side which reduced loadings at the prosthetic side [35]. Although the amputees necessarily put all the weight on the residual limb during walking, the magnitude of acceleration/deceleration of the center of mass could affect the ground reaction forces. Volume loss of the residual limb could also give rise to the reductions in pressures. It was reported in a previous study $[17,18]$ that prolonged walking led to residual limb volume loss, as the socket would act like a pump applying cyclic pressure to the limb which pushed fluid out of the residual limb. When a PTB socket is donned, some mechanical stresses, known as pre-stresses, are developed at the weight-bearing areas of the residual limb [36]. This is because PTB sockets have undercuts at these areas. The reduced residual limb volume lowers the pre-stresses and potentially reduces the stresses during walking [36].

There was a significant increase in tactile pressure threshold at the weightbearing popliteal depression area. This could be a result of the massage-like effect when the prosthetic socket repeatedly applying pressure onto the residual limb during walking $[25,26]$. Although there was a significant reduction in socket-limb interface pressure, the reduced tactile sensitivity could increase the risk of skin ulceration [37]. The decreased tactile sensitivity might also account for the walking instability because previous studies suggested that sensory degradation in elderly increased the likelihood of falls $[38,39]$. The changes in tactile pressure threshold at the other four regions were small and insignificant. Further study can investigate if the differences in soft tissue characteristics among regions, including soft tissue stiffness, underlying vascular and nerve structures, were responsible for these findings. 
One limitation of this study is that the duplicated liners might not exactly be the same as the subjects' currently used liners. One issue is that that the duration and temperature of heating as well as the degree of stretching over the duplication foam during the fabrication stage could be different, potentially affecting the mechanical properties of the liners. In addition, the currently used liners could have reduced thickness due to repeated compressions. Although the pressure measurements in all three time periods were performed with the same duplicated liner for each subject, eliminating the potential effects of liners when comparing the interface pressure among the three time periods, the measured pressure might not be exactly the same as those produced within the subjects’ own prostheses and liners.

Future studies can recruit subjects who are unable to walk long distances and investigate why they resist further walking. Changes in residual limb volume after LDW can be studied to investigate if they have any correlations with the reductions in socket-limb interface pressure. In addition, electromyography signal can be utilized to quantify muscle fatigue. Asymmetric gait pattern could be the leading cause of fatigue at the sound limb. Attempts should be made to refine prosthetic components and rehabilitation protocols aiming to restore the gait symmetry, and assess if it can improve LDW of lower-limb amputees.

\section{Conclusions}

This study investigated the effect of LDW on the socket-limb interface pressure, tactile sensitivity of the residual limb, and the self-reported subjective feedbacks from the amputees. It suggested that fatigue of the sound-side plantar flexors could be the main problem of long-distance walking. Muscle fatigue could hinder the walking stability, as the questionnaire revealed there were significant reductions in both the fatigue and walking stability scores. This may imply the 
importance of refining prosthetic components and rehabilitation protocols in reducing the muscle fatigue. After long-distance walking, there were significant reductions in socket-limb interface pressure and tactile sensitivity at the residual limb. These were in line with the subjective feedbacks of the subjects which revealed that the level of residual-limb discomfort and pain did not increase.

Declaration of Interests: The authors report no conflict of interest. This study was supported by an internal grant of The Hong Kong Polytechnic University.

\section{References}

1. Bragaru M, Dekker R, Geertzen J, Dijkstra PU. Amputees and sports: a systematic review. Sports Med 2011;41(9):721-40.

2. Burgess EM, Rappoport A. Physical fitness: a guide for individuals with lower limb loss. DIANE Publishing 1993.

3. Landi F, Russo A, Cesari M, Pahor M, Liperoti R, Danese P, Bernabei R, Onder G. Walking one hour or more per day prevented mortality among older persons: results from iISIRENTE study. Prev Med 2008;47:422-426.

4. Dudkiewicz I, Pisarenko B, Herman A, Heim M. Satisfaction rates amongst elderly amputees provided with a static prosthetic foot. Disabil Rehabil 2011;33(2122):1963-7.

5. Narang IC, Mathur BP, Singh P, Jape VS. Functional capabilities of lower limb amputees. Prosthet Orthot Int 1984;8:43-51.

6. Legro MW, Reiber GE, Czerniecki JM, Sangeorzan BJ. Recreational activities of lower-limb amputees with prostheses. J Rehabil Res Dev 2001;38:319-25. 
7. Modan M, Peles E, Halkin H, Nitzan H, Azaria M, Gitel S, et al. Increased cardiovascular disease mortality rates in traumatic lower limb amputees. Am J Cardiol. 1998;82:1242-7.

8. Naschitz JE, Lenger R. Why traumatic leg amputees are at increased risk for cardiovascular diseases. Q J Med 2008;101:251-9.

9. Yeung LF, Leung AK, Zhang M, Lee WC. Long-distance walking effects on trans-tibial amputees compensatory gait patterns and implications on prosthetic designs and training. Gait Posture 2012;35(2):328-33.

10. Sanders JE, Daly CH, Burgess EM. Interface shear stresses during ambulation with a below-knee prosthetic limb. J Rehab Res 1992;29:1-8.

11. Bader DL, Barnhill RL, Ryan TJ. Effect of externally applied skin surface forces on tissue vasculature. Arch Phys Med Rehabil 1986;67:807-11.

12. Mak FT, Zhang M, Boone DA. State-of-the-art research in lower-limb prosthetic biomechanics-socket interface. J Rehabil Res Dev 2001;38:161-74.

13. Zhang M, Lee WC. Quantifying the regional load-bearing ability of transtibial stumps. Prosthet Orthot Int 2006;30:25-34.

14. Voloshin AS, Mizrahi J, Verbitsky O, Isakov E. Dynamic loading on the human musculoskeletal system: effect of fatigue. Clin Biomech 1998;13:515-20.

15. Yoshino K, Motoshige T, Araki T, Matsuoka K. Effect of prolonged freewalking fatigue on gait and physiological rhythm. J Biomech 2004;37(8):1271-80.

16. Stolwijk NM, Duysens J, Louwerens JWK, Keijsers NLW. Plantar pressure changes after long-distance walking. Med Sci Sports Exerc 2010;42(12):2264-72.

17. Board WJ, Street GM, Caspers C. A comparison of trans-tibial amputee suction and vacuum socket conditions. Prosthet Orthot Int 2001;25(3):202-9. 
18. Beil TL, Street GM, Covey SJ. Interface pressures during ambulation using suction and vacuum-assisted prosthetic sockets. J Rehabil Res Dev 2002;39(6):693700.

19. Mak FT, Liu HW, Lee SY. Biomechanical assessment of below-knee residual limb tissue. J Rehabil Res Dev 1994;31:188-98.

20. Zhang M, Turner-Smith AR, Roberts VC, Tanner A. Frictional action at lower limb/prosthetic socket interface. Med Eng Phys 1996;18:207-14.

21. Dumbleton T, Buis AWP, McFadyen A, McHugh BF, McKay G, Murray KD, Sexton S. Dynamic interface pressure distributions of two transtibial prosthetic socket concepts. J Rehabil Res Dev 2009;46(3):405-16.

22. Sonck WA, Cockrell JL, Koepke GH. Effect of liner materials on interface pressure in below-knee amputees. Arch Phys Med Rehabil 1970;51:666-9.

23. Jia X, Suo S, Meng F, Wang R. Effects of alignment on interface pressure for transtibial amputee during walking. Disabil Rehabil Assist Technol 2008;3(6):339-43.

24. Mak AF, Zhang M, Tam EW. Biomechanics of pressure ulcer in body tissues interacting with external forces during locomotion. Annu Rev Biomed Eng 2010;12:29-53.

25. Lee WC, Zhang M, Mak AF. Regional differences in pain threshold and tolerance of the transtibial residual limb: including the effects of age and interface material. Arch Phy Med Rehabil 2005;86:641-9.

26. Ring C, Edwards L, Kavussanu M. Effects of isometric exercise on pain are mediated by blood pressure. Bio Psychol 2008;78(1):123-8. 
27. Dyck PJ, O’Brien PC, Kosanke JL, Gillen DA, Karnes JL. A 4,2,1 stepping algorithm for quick and accurate estimation of cutaneous sensation threshold. Neurology 1993;43(8):1508-12.

28. Nurse MA, Nigg BM. Quantifying a relationship between tactile and vibration sensitivity of the human foot with plantar pressure distributions during gait. Clin Biomech 1999;14:667-72.

29. McPoil TG, Cornwall MW. Plantar tactile sensory thresholds in healthy men and women. The Foot 2006;16:192-7.

30. Radcliff CW, Foort J. The patellar-tendon-bearing below-knee prosthesis.

Berkeley, CA: Biomechanics laboratory, University of California. 1961.

31. Buis AWP, Convery P. Calibration problems encountered while monitoring stump/socket interface pressures with force sensing resistors: techniques adopted to minimise inaccuracies. Prosthet Orthot Int 1997;21:179-82.

32. Polliack AA, Sieh RC, Craig DD, Landsberger S, McNeil DR, Ayyappa E. Scientific validation of two commercial pressure sensor systems for prosthetic socket fit. Prosthet Orthot Int 2000;24:63-73.

33. Legro MW, Reiber GD, Smith DG, Aguila M, Larsen J, Boone D. Prosthesis Evaluation Questionnaire for Persons With LowerLimb Amputations: Assessing Prosthesis-Related Quality of Life. Arch Phys Med Rehabil 1998;79:931-8.

34. Miller MD, Ferris DG. Measurement of subjective phenomena in primary care research: the Visual Analogue Scale. Fam Pract Res J 1993;13:15-24.

35. Lloyd CH, Stanhope SJ, Davis IS, Royer TD. Strength asymmetry and osteoarthritis risk factors in unilateral trans-tibial, amputee gait. Gait Posture 2010;32(3):296-300. 
36. Lee WCC, Zhang M, Jia XH, Cheng JTM. Finite element modeling of the contact interface between trans-tibial residual limb and prosthetic socket. Med Eng Phy 2004;26:655-62.

37. Dhruv NT, Niemi JB, Harry JD, Lipsitz LA, Collins JJ. Enhancing tactile sensation in older adults with electrical noise stimulation. Neuroreport 2002;13(5):597-600.

38. Lord SR, Clark RD, Webster IW. Postural stability and associated physiological factors in a population of aged persons. J Gerontol 1991;46(3):69-76.

39. Dhruv NT, Niemi JB, Harry JD, Lipsitz LA, Collins JJ. Enhancing tactile sensation in older adults with electrical noise stimulation. 
Figure 1: Attachment of Tekscan pressure sensors at the inner wall of the prosthetic liner.

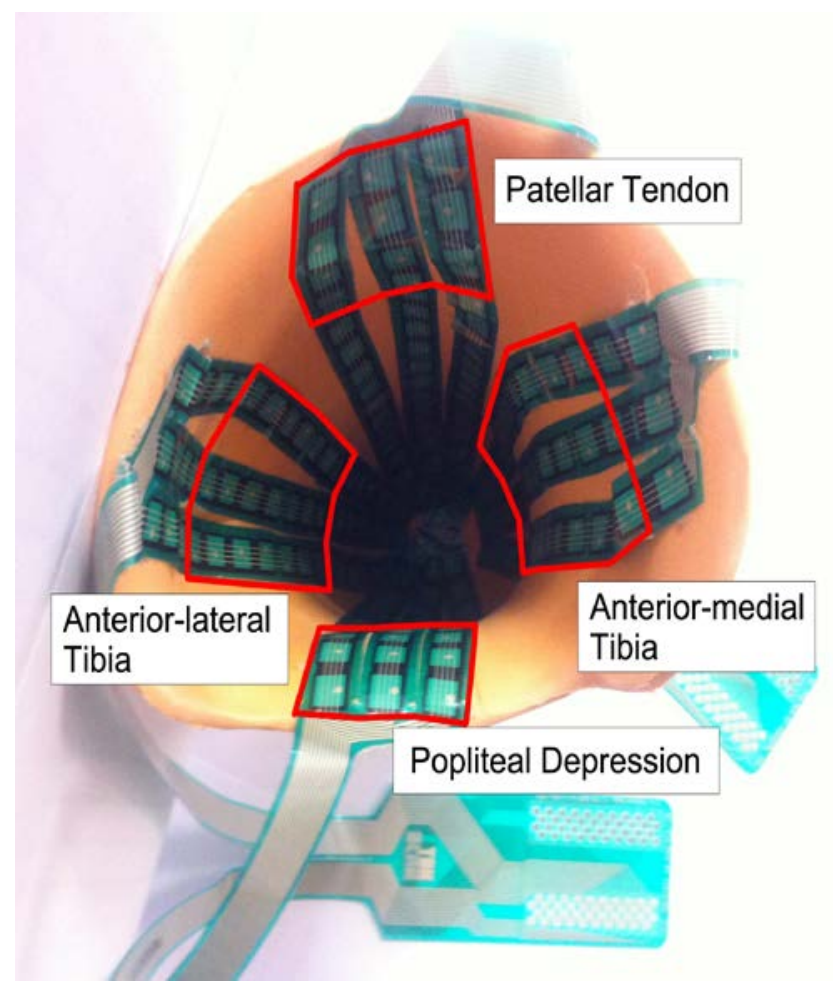


Figure 2: Dynamic socket-limb interface pressure in the stance phase of the gait cycle averaged across subjects.
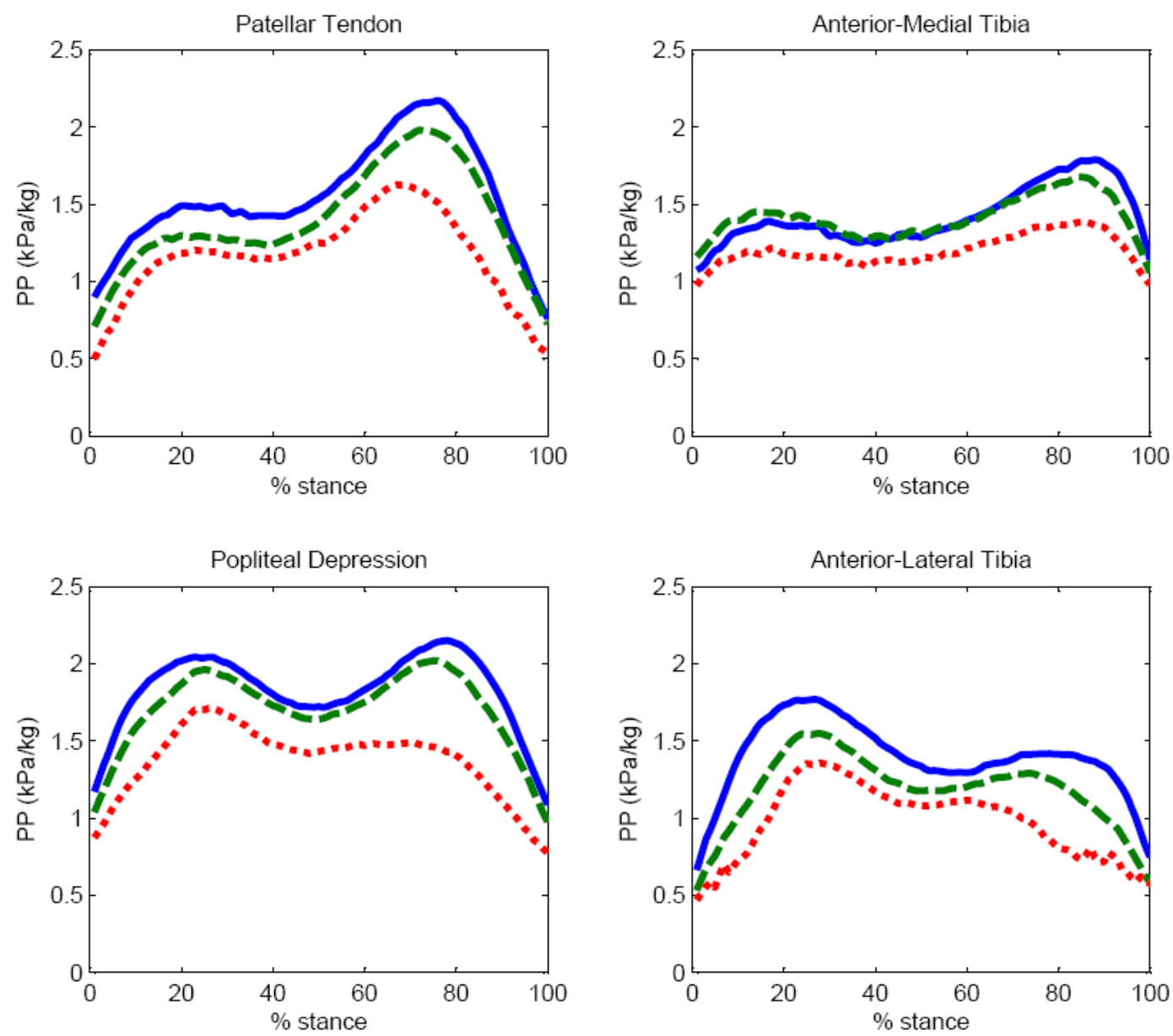

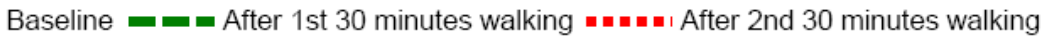


Table 1: Characteristics of participated amputee subjects.

\begin{tabular}{|l|l|l|l|l|l|l|l|l|}
\hline $\begin{array}{l}\text { Amputee } \\
\text { participants }\end{array}$ & Gender & Age (years) & $\begin{array}{l}\text { Height } \\
(\mathrm{cm})\end{array}$ & $\begin{array}{l}\text { Body mass } \\
(\mathrm{kg})\end{array}$ & $\begin{array}{l}\text { Time since } \\
\text { amputation } \\
(\text { years })\end{array}$ & $\begin{array}{l}\text { Amputated } \\
\text { limb }\end{array}$ & $\begin{array}{l}\text { Type of } \\
\text { prosthetic } \\
\text { foot }\end{array}$ & $\begin{array}{l}\text { Reason for } \\
\text { amputation }\end{array}$ \\
\hline A & M & 61 & 167.5 & 65.6 & 39 & L & SACH & Traumatic \\
\hline B & M & 47 & 170 & 74.6 & 9 & R & SACH & Traumatic \\
\hline C & M & 54 & 166.5 & 79 & 45 & L & SACH & Traumatic \\
\hline D & M & 62 & 175.5 & 75 & 22 & R & SACH & Traumatic \\
\hline E & M & 39 & 167 & 75.5 & 17 & R & SACH & Vascular \\
\hline Mean (SD) & & $52.6(4.3)$ & $169.3(1.7)$ & $73.9(2.2)$ & $26.4(6.8)$ & & & \\
\hline
\end{tabular}


Table 2: The six questions used in the questionnaire survey. Each question was scored based on the distance of the marking from the left anchor.

Q.1: Rate the fitting of your prosthesis.

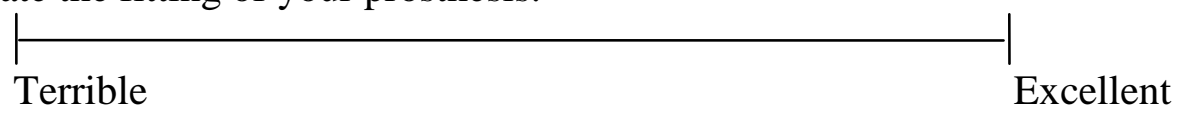

Q.2: Rate the conditions (including temperature, humidity, and texture) of the socket on your residual limb.

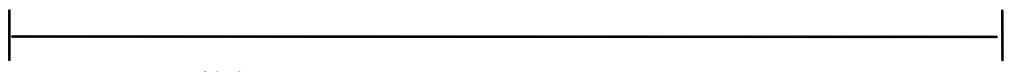

$\begin{array}{ll}\text { Worse possible } & \text { Best possible }\end{array}$

Q.3: Rate how well you can balance with your prosthesis during walking.

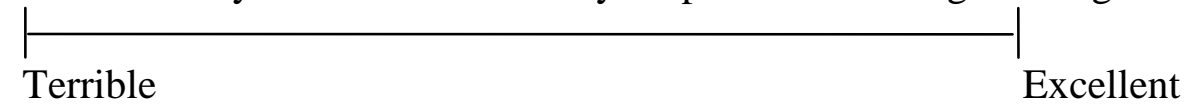

Q.4: Rate the comfort of residual limb while using your prosthesis.

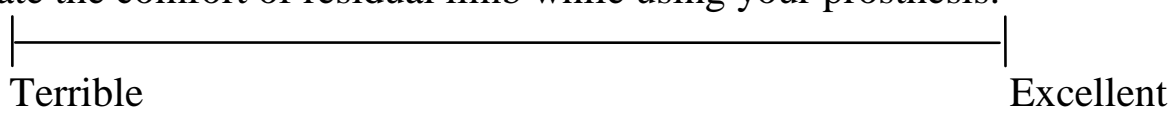

Q.5: Rate the intensity of pain you felt at the residual limb (if any).

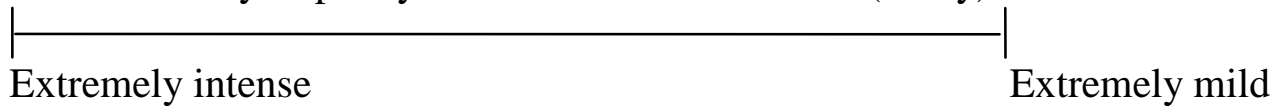

Q.6: Rate the intensity of fatigue you felt in the lower-limb (if any).

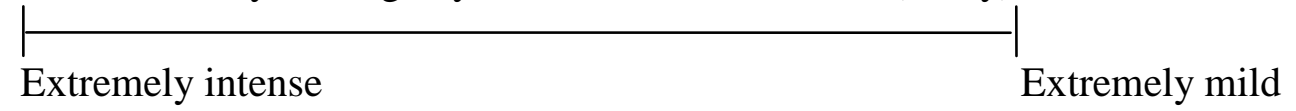

Please point at the location(s) of your residual limb where the highest intensity of fatigue was perceived. 
Table 3: Mean (SD) of the walking speed and peak socket-limb interface pressure.

\begin{tabular}{|c|c|c|c|}
\hline & Baseline & $\begin{array}{c}\text { After 30-minute } \\
\text { walking }\end{array}$ & $\begin{array}{c}\text { After 1-hour } \\
\text { walking }\end{array}$ \\
\hline $\begin{array}{c}\text { Self-selected comfortable } \\
\text { walking speeds (m/s) }\end{array}$ & $1.11(0.10)$ & $1.10(0.08)$ & $1.15(0.12)$ \\
\hline \multicolumn{4}{|c|}{ Peak Interface Pressure $(\mathrm{kPa} / \mathrm{kg})$} \\
\hline Patellar tendon & $2.39(1.70)$ & $2.24(1.43)$ & $1.95(1.49)$ \\
\hline Popliteal depression & $2.22(1.32)$ & $2.19(1.17)$ & $1.80(1.37) *$ \\
\hline Anterior-medial tibia & $1.90(1.25)$ & $1.81(0.93)$ & $1.51(1.27)$ \\
\hline Anterior-lateral tibia & $1.82(0.88)$ & $1.61(0.86)$ & $1.44(1.02) *$ \\
\hline
\end{tabular}

* Statistical significantly different when compared with the baseline value. 
Table 4: Mean (SD) of the residual limb tactile threshold and the subjective feedback scores.

\begin{tabular}{|c|c|c|}
\hline & Baseline & 1-hour walking \\
\hline \multicolumn{3}{|c|}{ Tactile Pressure Threshold (log force) } \\
\hline Patellar Tendon & $4.1(0.8)$ & $4.3(0.9)$ \\
\hline Popliteal Depression & $2.5(0.6)$ & $3.1(0.5) *$ \\
\hline Anterior-medial Tibia & $3.0(0.8)$ & $3.3(0.6)$ \\
\hline Anterior-lateral Tibia & $3.1(0.9)$ & $3.3(0.3)$ \\
\hline \multicolumn{3}{|c|}{ Subjective Feedback Scores $(\mathrm{cm})$} \\
\hline Socket fitting score (Q1) & $8.6(0.4)$ & $7.8(1.0) *$ \\
\hline Interface conditions score (Q2) & $5.7(3.3)$ & $5.3(3.2)$ \\
\hline Dynamic stability score (Q3) & $8.6(0.1)$ & $7.8(0.1) *$ \\
\hline Residual-limb comfort score (Q4) & $8.8(0.1)$ & $8.3(0.4)$ \\
\hline Residual-limb pain feeling (Q5) & $7.3(3.3)$ & $7.1(2.3)$ \\
\hline Lower-limb muscle fatigue (Q6) & $7.0(0.7)$ & $2.1(0.8) *$ \\
\hline
\end{tabular}

* Statistical significantly different when compared with the baseline value. 